\title{
El estudio del control social en Foucault: lectura y discusión en el aula desde la metodología participativa y la formación reflexiva.
}

\author{
Social control in Foucault: reading and discussion \\ in the classroom from the participative methodology \\ and reflective practice.
}

Jorge Correcher Mira

Profesor ayudante doctor, Departamento de Derecho penal.

Facultad de Derecho.

Universitat de València.

E-mail: jorge.correcher@uv.es

\begin{abstract}
Resumen: El presente artículo recoge los puntos básicos sobre la actividad de evaluación continua desarrollada en la asignatura Derecho penitenciario, obligatoria del Doble Grado en Derecho y Criminología, impartido en la Universitat de València. La propuesta consiste en la lectura dirigida del libro Vigilar y castigar, escrito por Michel Foucault, así como en la adaptación de las tesis del autor francés al contexto propio de la revolución digital por el filósofo coreano Byung Chul-Han en su libro La sociedad de la transparencia.
\end{abstract}

A partir de las lecturas propuestas, se organizaron en el aula una serie de sesiones presenciales, siguiendo distintas metodologías de enseñanza participativa, para discutir las principales tesis de estos autores, poniéndolas en relación con el estudio de los instrumentos de control social propios del sistema penal, como lo es el Derecho penitenciario. Se pretende así profundizar en aspectos transversales a la asignatura objeto de estudio, a partir de un proceso reflexivo de formación, ofreciendo al alumnado una aproximación crítica al estudio del control social, indispensable para garantizar su formación como juristas y criminólogos.

Palabras clave: Metodología participativa, formación reflexiva, debate, grupos de discusión, control social, Derecho penitenciario 
Abstract: This article summarizes the main points related to the continuous evaluation activity developed in the subject Penal Law, compulsory in the ungraduated course Law and Criminology, University of Valencia. The proposal is based on the supervised reading of the book Survey and Punish, written by Michel Foucault, and also in the adaptation of his thesis to the digital revolution context by the Corian philosopher Byung Chul-Han in the book The Transparency Society.

According to the proposed readings, classroom sessions were organized, following participatory methodologies, with the purpose of discussing the main ideas of these authors, related to social control institutions in the penitentiary law. The idea was looking into cross features of the subject, following a reflective practice process in the classroom, giving to the students a critical approach to social control study, necessary to its development as legal experts and criminologists.

Keywords: Participative methodology, reflective practice, deliberation, discussion group, social control, penal law.

\title{
El estudio del control social en Foucault: lectura y discusión en el aula
}

desde la metodología participativa y la formación reflexiva.

\author{
Social control in Foucault: reading and discussion in the classroom from the \\ participative methodology and reflective practice.
}

\section{Notas preliminares}

La obra de Michel Foucault supone una de las más valiosas aportaciones a la filosofía contemporánea, imprescindible para contextualizar desde una perspectiva crítica las distintas tensiones y conflictos político-culturales derivados de la posmodernidad ${ }^{1}$. Desde el punto de vista de las relaciones de poder y el estudio del control social, su libro más significativo es Vigilar y castigar, primera edición en francés publicada en $1975^{2}$, donde desarrolla a los largo de sus cuatro capítulos ${ }^{3}$ una aproximación funcional a las instituciones de control social. En este libro, presenta conceptos básicos para el análisis crítico del sistema de justicia penal, como lo son las nociones de

\footnotetext{
1 Para una reciente aproximación al pensamiento y figura de Michel Foucault, puede destacarse la aportación de Villacañas, J.L. y Castro, R. (2018) Foucault y la historia de la filosofía, Dado, Madrid.

${ }^{2}$ La edición seguida en castellano ha sido la siguiente: Foucault, M. (2009) Vigilar y castigar, Siglo XXI, Madrid.

${ }^{3}$ Titulados respectivamente: Suplicio, Castigo, Disciplina y Prisión.
} 
ilegalismo o panóptico, de especial trascendencia para entender los fundamentos del control social formal, donde el Derecho penitenciario ocupa un lugar de preeminencia. Mediante el estudio de este autor, y la aplicación de sus postulados al estudio del control social, se pretende reforzar el carácter crítico de la docencia universitaria. Siguiendo a IMBERNÓN (2013), “aunque la universidad sea una institución al servicio de la sociedad no puede limitarse únicamente a transmitir la cultura que ella ha generado y genera, sino que ha de analizar críticamente lo que hay dentro y fuera de sus fronteras para reconocerse en una nueva universidad en la sociedad de la información y el conocimiento" (p. 17).

De acuerdo con lo expuesto, se consideró por el profesorado responsable de la asignatura $^{4}$ el interés que podría tener la lectura dirigida de esta obra dentro del proceso de evaluación continua de la asignatura Derecho penitenciario, especialmente por el carácter transversal que ésta debe tener dentro del Doble Grado en Derecho y Criminología. Sobre esta cuestión, se identificó la importancia de esta lectura para reforzar el cumplimiento de los siguientes resultados de aprendizaje previstos en la guía docente de la asignatura (resultados 1, 3 y 4):

Identificar los efectos criminógenos de la prisión y la problemática específica de las personas privadas de libertad.

Conocer y analizar la problemática de la pena de prisión y su significado en el sistema sancionador.

Analizar y valorar los efectos de la resocialización como fin del sistema penitenciario.

Asimismo, si bien la lectura y debate de Vigilar y castigar supondría el grueso de las sesiones previstas, se complementaría su lectura con la adaptación de las tesis del pensamiento de Foucault al contexto propio de la revolución digital y el big data, presentada por el filósofo coreano Byung Chul-Han en su libro La sociedad de la transparencia. Se pretendía de este modo ofrecer al alumnado, una vez interiorizadas las ideas centrales de Foucault, una interpretación renovada de sus postulados, en tanto que adaptada a las nuevas perspectivas en el estudio de las relaciones de control y poder derivadas de una realidad que pueden identificar como propia. De este modo, se pretende dotar a la actividad de un enfoque profundo, entendido como aproximación

\footnotetext{
${ }^{4}$ Además del autor de este artículo, el Prof. Dr. Javier Mira Benavent, Profesor Titular de Derecho penal
} de la Universitat de València. 
que permita a los estudiantes asumir un rol activo en su formación, actuando con el deseo de comprender los puntos principales del nuevo contenido, estableciendo conexiones y extrayendo conclusiones (MEDINA y JARAUTA, 2013, p.143).

\section{Presupuestos metodológicos: innovación desde la participación y la formación reflexiva}

Para el desarrollo de la evaluación continua a partir del estudio de las obras reseñadas en el punto anterior, se ha partido del uso de metodologías participativas que garanticen una formación reflexiva en el alumnado y profesorado universitario. Partiendo de la definición de LÓPEZ (2005), la metodología participativa "se basa en los procesos de intercambio -de conocimientos, experiencias, vivencias y sentimientos, etc.- y en la construcción colectiva de conocimientos que se propicia entre los sujetos que componen el grupo" (p. 57). Desde esta postura, se entiende como parte del capital educativo la posibilidad de plantear metodologías grupales donde prime la participación de los/las estudiantes, estableciendo así entre todos los agentes del aula un proceso de formación o práctica reflexiva. Se entiende ésta como:

La práctica reflexiva es la base de la profesionalidad efectiva en cualquier campo, incluyendo la enseñanza universitaria. La práctica reflexiva puede estimularse y dirigirse formalmente como "aprendizaje en la acción" que consiste en buscar de forma sistemática el progreso del propio ejercicio docente y en asegurarse que los cambios se efectúan en la dirección correcta, en concreto, que sus alumnos están aprendiendo ahora mejor de lo que solían. El objetivo del aprendizaje-acción es la enseñanza de la persona a sí misma de modo que no sólo abarca el aprendizaje del estudiante, ni siquiera el aprendizaje sobre la enseñanza, sino el aprendizaje sobre uno mismo como profesor y la utilización de la reflexión para llegar a ser un profesor mejor. (BIGGS, 2010, p. 25).

Partiendo de las dos premisas metodológicas expuestas, se pretende dotar al estudio de las obras reseñadas de un alcance crítico, no sólo desde la propia materia objeto de estudio, sino en relación con las propias prácticas educativas de los estudios en ciencias jurídicas ${ }^{5}$. Para ello, partiendo de lo que se ha definido como metodología

\footnotetext{
${ }^{5}$ Un ejemplo de crítica a la enseñanza del Derecho, especialmente con posterioridad a la implantación del Espacio Europeo de Educación Superior, puede verse en: Quintero Olivares, G. (2010): La enseñanza del Derecho en la encrucijada. Derecho académico, docencia universitaria y mundo profesional, CivitasThomson Reuters, Navarra.
} 
participativa se busca fomentar la estimulación y la motivación al trabajo, el aumento de la creatividad, el permitir analizar puntos de vista diferentes, el desarrollar la capacidad de cooperación y favorecer el intercambio de experiencias (IMBERNÓN, 2013, pp. 29-30). En este sentido, como se verá en el punto 3 cuando se describa el desarrollo de la actividad en el aula, se han seguido técnicas participativas como el grupo de discusión y el debate, para así reforzar la oralidad y capacidad argumentativa de los/las estudiantes ${ }^{6}$. Se ha seguido esta orientación en tanto que se reconoce la importancia de estas técnicas para el estudio de las ciencias jurídicas, de forma que se puedan potenciar competencias relacionadas con la capacidad de argumentación jurídica y la habilidad de defensa oral de un discurso razonado, básicas para un jurista penal (VIANA, 2017, p. 312).

\section{Descripción de la actividad a desarrollar en el aula}

En el siguiente apartado, se describirá la actividad propuesta partiendo de los presupuestos metodológicos expuestos en el punto previo. Para ello, se detallarán en primera instancia, además de los datos básicos de la asignatura, las premisas establecidas en la guía docente de la asignatura para la evaluación continua. Partiendo de éstas, se preparó por el profesorado un anexo específico para el proceso de evaluación continua, concretando así los criterios que servirían de base para la valoración cualitativa de la actividad descrita en este artículo. Expuestas estas consideraciones, se pasará a exponer el desarrollo material de las sesiones presenciales en el aula, a partir de los presupuestos metodológicos desarrollados en el punto anterior.

\footnotetext{
${ }^{6}$ En este punto, se parte de los resultados obtenidos en el proyecto de innovación educativa y renovación de metodologías docentes titulado "Nuevos métodos de evaluación en las estrategias didácticas de aprendizaje colaborativo: oralidad y argumentación en el ámbito del Derecho penal" (código UVSFPIE_RMD16-418741), concedido por el Vicerrectorado de Formación y Calidad Educativa de la Universitat de València, coordinado por Clara Viana Ballester, Profesora contratada doctora de Derecho penal, Universitat de València.
} 


\subsection{Pautas organizativas a partir de la guía docente}

Asignatura: Derecho penitenciario

Código: 35064

Titulación: Doble grado en Derecho y Criminología

Créditos ECTS: 6

Carácter: Obligatorio

Grupo: DV

Idioma: Valenciano

Profesorado Departamento Derecho penal 2017-2018:

Prof. Dr. Javier Mira Benavent, Profesor Titular, Derecho penal

Prof. Dr. Jorge Correcher Mira, Profesor Ayudante doctor, Derecho penal

De acuerdo con la guía docente de la asignatura Derecho penitenciario, corresponde a la evaluación continua el 30\% de la puntuación total de la asignatura. Dado que no se especificaba nada más, se decidió adjuntar a los estudiantes en aula virtual una guía del proceso de evaluación continua. En dicha guía, se concretaba en el primer apartado la puntuación asignada a cada una de las actividades a realizar, redactándose de la siguiente manera $^{7}$ :

a) Asistencia activa a clase en las sesiones presenciales de trabajo y discusión: $10 \%$. La referencia a asistencia activa significa que para valorar este $10 \%$ no sería suficiente con encontrarse físicamente en el aula, sino que será necesaria una participación que muestre el trabajo de los materiales.

b) Trabajo de análisis crítico sobre las lecturas trabajadas en las sesiones presenciales: $20 \%$.

Como paso previo a la exposición de ambas dinámicas, debe matizarse que, pese a estar dicho documento disponible en el aula virtual de la asignatura, para facilitar la posible aclaración de dudas por parte de los/las estudiantes fueron realizadas en la primera sesión del cuatrimestre una serie de concreciones durante la exposición del proceso de evaluación de la asignatura. De este modo, la primera sesión presencial podría ser utilizada de forma íntegra, mejorando de este modo el denominado efecto primacía (IMBERNÓN MUÑOZ, 2013, p. 24). Además, permitía tener un primer contacto, previo al inicio de las sesiones presenciales, con los/las estudiantes, aclarando sus posibles dudas sobre las dinámicas a desarrollar en clase.

\footnotetext{
${ }^{7}$ La docencia, así como los materiales suministrados para el desarrollo de ésta han sido redactados originalmente en valenciano por ser la lengua en que se impartía la asignatura. Por razones prácticas, las citas literales a los materiales utilizadas en este artículo serán traducidas al castellano.
} 
Para cada uno de los apartados objeto de puntuación de la asignatura ${ }^{8}$, se redactó en el anexo de la evaluación continua una serie de premisas para orientar a los/las estudiantes. Por un lado, en el punto 3 "Metodología de trabajo de las sesiones presenciales de discusión" se establecen las siguientes directrices para organizar el trabajo en el aula:

“A partir de la lectura de los libros que serán indicados previamente a cada una de las sesiones presenciales, será utilizado el tiempo de clase para comentar y discutir, sea de forma individual o grupal en función de la temática, los aspectos más relevantes de las lecturas recomendadas. En este sentido, el profesor sugerirá una serie de cuestiones nucleares de las lecturas, relacionadas con la idea de control social, la filosofía del castigo, la legitimación de los modelos penales...En todo caso, para que el debate no sea autorreferencial, es tratará de relacionar siempre que la temática lo permita- la discusión de las lecturas con cuestiones de actualidad. Será indispensable la lectura previa de los materiales”.

Para la organización de estas sesiones presenciales de discusión sobre los materiales, se facilitó a los alumnos un cronograma, donde se determinaban los días reservados para las sesiones de evaluación continua. Concretamente, se dedicaron 4 sesiones de clase, en horario de 12:30 a 14:30. Por la extensión de la obra, los tres primeros días fueron dedicados a comentar los capítulos del libro Vigilar y castigar, mientras que la última sesión se dedicaría al libro La sociedad de la transparencia, con una extensión mucho más modesta.

\begin{tabular}{|l|l|}
\hline Martes 13 de febrero & Vigilar y castigar. SUPLICIO \\
\hline Martes 6 de marzo & Vigilar y castigar. CASTIGO \\
\hline Martes 10 de abril & $\begin{array}{l}\text { Vigilar y castigar. DISCIPLINA y } \\
\text { PRISIÓN }\end{array}$ \\
\hline Martes 24 de abril & La Sociedad de la transparencia. \\
\hline
\end{tabular}

En el anexo de evaluación continua facilitado a los/las estudiantes, también se incluyó un apartado orientativo sobre las pautas para la realización del trabajo de análisis crítico en que consistía el $20 \%$ de la puntuación. Bajo la rúbrica "Metodología para la realización del trabajo de análisis crítico", el apartado 4 del anexo dispone:

\footnotetext{
${ }^{8}$ Recuérdese, $10 \%$ para la asistencia activa a clase en las sesiones presenciales de trabajo y discusión, así como $20 \%$ por la realización de un trabajo de análisis crítico sobre las lecturas trabajadas en las sesiones presenciales.
} 
"Este trabajo no supone realizar un resumen de los libros trabajados en el curso. A partir de su lectura, así como de la discusión en las sesiones presenciales, se presentará un ensayo o trabajo de análisis crítico a partir de cualquiera de las cuestiones tratadas en las sesiones presenciales. Este análisis debe poner en relación los conceptos y argumentos estudiados en los libros con una aproximación crítica al sistema de justicia penal español, sea a la propia realidad penitenciaria, el discurso político-criminal actual, las últimas reformas en materia penal, la limitación de los derechos de libertad y la criminalización de la disidencia mediante los delitos de opinión en las redes sociales (...) por tanto, para la realización del trabajo se demostrarán los conocimientos adquiridos sobre los autores estudiados durante el cuatrimestre, desarrollando asimismo la capacidad crítica mediante la aplicación de sus postulados a las instituciones de control social estudiadas a lo largo de la formación como juristas y criminólogos".

En la realización de este trabajo, podría reconocerse la denominada por Humboldt como forschendes Lernen (aprendizaje en modo de investigación), en tanto que se pretende la realización por parte del alumnado de un trabajo de investigación que, sin aspirar a ofrecer una serie de propuestas o soluciones como podría darse en una investigación de postgrado, muestren la necesidad de plantear las cuestiones problemáticas o controvertidas propuestas por el docente -por ejemplo, la idea de control social analizada en las lecturas- como una discusión inacabada. En consecuencia, este aprendizaje en modo de investigación permite, de acuerdo con ELTON (2010), "plantear interrogantes de una forma que raramente se observa en el aprendizaje tradicional, en el que las personas que aprenden reaccionan a la información proporcionada por el docente. Por tanto, el principal papel de los docentes consiste en comprender pedagógicamente cómo pueden facilitar este aprendizaje a través de la interrogación” (p. 149).

\subsection{Sesiones presenciales de trabajo: grupos de discusión y debate}

Para cada una de las sesiones de trabajo sobre el libro Vigilar y Castigar, se les pedía previamente a los alumnos que hubieran leído el capítulo correspondiente, teniendo éstos una extensión media de unas 80 páginas. Como puede apreciarse en el cronograma, el espacio temporal entre las sesiones presenciales (dependiendo de la periodificación, de 2 a 4 semanas), permitía que fuera una extensión asumible por el alumnado. En estas sesiones, se desarrollaba la clase a partir de la siguiente estructura: 
- Inicio de la clase con una breve introducción o recopilación de ideas sobre el capítulo en cuestión, siendo ésta realizada en unos 5-10 minutos por parte del profesor.

- Posteriormente, se les repartía a los/las estudiantes un documento con 8 citas significativas sobre el capítulo que previamente habían leído, acompañando esas citas del autor con unas preguntas escritas en negrita que trataban de contextualizar el contenido del libro con el sistema de justicia penal actual, la transformación de los mecanismos de control social formal e informal, el auge de las nuevas tecnologías en la revolución digital...En resumen, cuestiones que permiten observar la utilidad del pensamiento de Foucault para analizar críticamente el sistema de relaciones de poder y control resultante de los Estados dominados por la hegemonía del pensamiento neoliberal.

Se ofrecen a continuación dos de las citas significativas, acompañadas de sus correspondientes preguntas, para que pueda así apreciarse cómo se pretendía contribuir con éstas a la formación del estudiante:

a) "el poder disciplinario, gracias a la vigilancia jerarquizada, se convierte en un sistema integrado vinculado del interior a la economía y a los fines del dispositivo en que se ejerce. Se organiza también como un poder múltiple, automático y anónimo; porque si es cierto que la vigilancia reposa sobre individuos, su funcionamiento es el de un sistema de relaciones de arriba abajo, pero también hasta cierto punto de abajo arriba y lateralmente. Este sistema hace que resista el conjunto, y lo atraviesa íntegramente por efectos de poder que se apoyan unos sobre otros: vigilantes perpetuamente vigilados" (Foucault, 2009, pp. 181-182) Desde esta visión funcional del poder disciplinario, ¿cómo puede reconocerse la importancia que otorga Foucault a agentes externos al sistema de justicia penal, más propios del control social informal, como son la familia, sistema educativo, grupo religioso, lugar de trabajo...?

b) "detrás del infractor al cual la investigación de los hechos puede atribuir la responsabilidad de un delito se perfila el carácter delincuente cuya lenta formación se ha demostrado por una investigación biográfica. La introducción de lo biográfico es importante en la historia de la penalidad. Porque hace existir al criminal antes del crimen (...) como correlato de la justicia penal, tenemos, sin duda, al infractor; pero el correlato del aparato penitenciario es otro; es el 
delincuente, unidad biográfica, núcleo de peligrosidad, representante de un tipo de anomalía. Y si es cierto que a la detención privativa de libertad que había definido el derecho, ha agregado la prisión el suplemento de la penitenciaria, ésta a su vez ha introducido a un personaje de sobra, que se ha deslizado entre el que la ley condena y el que ejecuta esta ley” (Foucault, 2009, p. 258) ¿Cómo afecta la creación de este personaje (categoría del delincuente) al principio a la presunción de inocencia, especialmente considerando la importancia en su desarrollo de variantes sociales o culturales?

- Como puede apreciarse a partir de la lectura de este ejemplo, se pretende fomentar la existencia de lo que se ha denominado como conflicto cognitivo en el aula, puesto que se critican cuestiones que parecen darse por establecidas en el estudio del sistema de justicia penal o del control social. En este sentido, se entiende este instrumento - conflicto cognitivo- como situaciones que generan un conflicto entre lo que los alumnos creen saber y un conocimiento que contradice esas creencias o un conflicto entre los propios saberes disciplinares (MEDINA y JARAUTA, 2013, p. 143).

- Repartido este documento, se le daba a los/las estudiantes un tiempo medio de 20-25 minutos para la lectura de todas las citas significativas del capítulo, seleccionadas por el profesor para trabajar durante la clase.

- Pasado ese tiempo, se organizaba a los alumnos en 8 grupos de entre 3 y 4 estudiantes. Dado que el total de matriculados eran 33, el número de componentes dependía de la asistencia a las sesiones presenciales, pero dado que la mínima fue de 24 en la primera sesión, siempre se pudieron formar 8 grupos, que por lo que se verá a continuación era indispensable para la dinámica de clase. Estos grupos se organizan como pequeños grupos de discusión, considerando esta metodología participativa adecuada, en tanto que permite apreciar la intervención de varios procesos, como el ordenamiento de los propios pensamientos, exposición en público, análisis crítico, etc. (LÓPEZ, 2005, p. 155).

- A cada uno de los 8 grupos, se les asignaban 2 de las 8 citas significativas recogidas en el documento correspondiente a la sesión de clase, de forma que para cada par de citas siempre habría dos grupos a los que éstas se les habría 
asignado. En la medida de lo posible, se intentó garantizar la paridad en los grupos.

- Asignadas dos citas significativas para cada grupo, se les deja a los/las estudiantes 20-25 minutos para discutir las citas, poniéndolas en relación con los aspectos señalados por el profesor en las preguntas de reflexión incluidas al final de cada cita. Cada uno de los grupos debía anotar en un folio sus respuestas o reflexiones sobre las preguntas, utilizando también este documento como control de asistencia. Se pretende así que la división en pequeños grupos permita apreciar una interacción cooperativa, de forma que pueda existir una correlación positiva entre los/las estudiantes que lo componen para conseguir un objetivo común, siendo las recompensas o refuerzos que logran los individuos directamente proporcionales a la calidad del trabajo en grupo.

- Pasado el máximo de 25 minutos, se inicia en clase el comentario de cada una de las citas y sus pertinentes preguntas, siendo conducido por el profesor, y participando como hilo conductor en el debate cada uno de los grupos a los que les había tocado discutir sobre la pregunta en cuestión. Se partiría aquí de la técnica participativa conocida como discusión dirigida o debate, permitiendo extrapolar las pequeñas discusiones previas a un debate articulado en el conjunto de la clase, estimulando de este modo la participación y el intercambio de opiniones (LÓPEZ, 2005, p. 158).

- En este punto, la enseñanza reflexiva supone que la comprensión del propio contenido del texto transcurra en paralelo al entendimiento de los conceptos presentados por el profesor. De acuerdo con Imbernón et al. (2010) "una de las finalidades de la enseñanza universitaria es que el alumnado 'comprenda' aquellos aspectos de los ámbitos disciplinares que el docente trata de vehicular. El proceso de comprensión es similar a la interpretación de un texto. Cuando el alumnado trata de comprender algunos de los aspectos tratados en el aula, se aproxima a ellos con una serie de significados, creencias y expectativas previas, que interaccionan con el significado que el docente intenta comunicar, de manera que este contenido le resulte más comprensible o significativo" (p. 32).

- Finalizado el turno de intervención de cada uno de los grupos, lo más habitual era que participaran estudiantes de otros, puesto que el conjunto de los/las 
estudiantes tenían conocimiento de todas las preguntas, puesto que las habían leído al inicio de la sesión.

Para la sesión de trabajo sobre el libro La sociedad de la transparencia, la metodología fue distinta, motivada también por las circunstancias que permitían un giro en la dinámica. Sin entrar en un análisis detallado de esta obra, puede simplemente señalarse que supone una renovación en parte del pensamiento de Foucault, en tanto que amplía los espacios de control social descritos por el pensador francés al ámbito de las redes sociales, el big data...Por ello, dada la aplicación práctica de este fenómeno en la actualidad, se decidió organizar un seminario en clase sobre manifestaciones actuales de los postulados de este autor, siendo también preceptivo que los/las estudiantes hubieran leído previamente la obra. Este seminario fue introducido por los profesores de la asignatura, siendo impartido por la Prof. Marina Sancho López, personal investigador en formación del Departamento de Derecho civil de la Universitat de València, especializada en el big data y los límites de la privacidad y Andreu Moreno Tarín, abogado penalista colegiado en el Ilustre Colegio de Abogados de València, consultor del comité de expertos del grupo parlamentario confederal de la izquierda unitaria europea, dentro del Parlamento Europeo, relativo al estudio del discurso del odio y los límites a la libertad de expresión en España.

De este modo, se pretendía mostrar a los estudiantes cómo puede afectar en la práctica, en la aplicación del Derecho penal, la realidad presentada en el libro La sociedad de la transparencia, deudora en sus fundamentos de las tesis presentadas por Foucault en Vigilar y castigar. La exposición de los invitados no siguió un formato clásico de seminario-ponencia, en tanto que durante su presentación, de unos 20 minutos cada uno, permitieron a los/las estudiantes formular dudas, observaciones, e incluso rebatir parte del discurso de su intervención. En estas interpelaciones, podía observarse que habían asimilado la lectura de la obra, enriqueciendo el debate y las aportaciones de los invitados. En este sentido, se pudo obtener como corolario de la formación reflexiva propuesta en esta actividad una importante asunción de los procesos de interacción comunicativa por parte de los/las estudiantes, la cual venía acompañada por un uso adecuado de los conceptos clave estudiados en ambos autores, en relación con los resultados de aprendizaje recogidos en la guía docente de la asignatura. 


\section{Conclusión}

Como se ha expuesto en estas páginas, la actividad de evaluación continua planteada a partir de las lecturas propuestas partía de la consideración de la enseñanza universitaria como un continuo proceso de aprendizaje crítico para todos los actores del aula. Esta vocación crítica viene referida tanto a los contenidos objeto de estudio, como respecto de la metodología utilizada por el docente, y como el alumnado debe verse implicado en ésta. Para armonizar esta complementariedad, se ha seguido lo dispuesto por la práctica o formación reflexiva, considerando que los debates y discusiones planteados en las sesiones presenciales tuvieran un alcance transformador, no sólo en el estudio y asimilación de los contenidos por parte de los/las estudiantes, sino también en la propia formación docente del profesor. Para ello, ha sido especialmente significativo el estudio de las tesis de Michel Foucault, en tanto que sus ideas sobre control social desde una perspectiva crítica favorecen la posibilidad de innovar en el aula mediante metodologías participativas como el debate o el grupo de discusión. En este sentido, la posibilidad de construir discursos críticos desde sus posiciones en relación con el estado actual del sistema penal español, permitía el fomento del conflicto cognitivo entre los/las estudiantes, favoreciendo de este modo su discusión y aprendizaje. A la espera de recibir los datos de las encuestas formuladas al alumnado respecto del profesor de la asignatura $^{9}$, los resultados obtenidos por los/las estudiantes ${ }^{10}$ han sido muy positivos, siendo además gratificante su implicación y compromiso durante el desarrollo de la actividad.

\section{Bibliografía}

Biggs, J. (2010) Calidad del aprendizaje universitario, Narcea, Madrid.

Elton, L. (2008) "El saber y el vínculo entre la investigación y la docencia", en Para una transformación de la universidad. Nuevas relaciones entre investigación, saber y docencia (Barnett ed.), Octaedro, Granada.

Foucault, M. (2009) Vigilar y castigar, Siglo XXI, Madrid.

Imbernón Muñoz, F. (2013) "Referentes para una didáctica reflexiva en la universidad”, en Enseñanza y aprendizaje en la educación superior, Síntesis, Madrid.

\footnotetext{
${ }^{9}$ Coordinadas y gestionadas en la Universitat de València por la Unidad de Calidad.

${ }^{10}$ Los 33 estudiantes matriculados en la asignatura siguieron de forma constante la evaluación continua, siendo aprobada también por todo el alumnado.
} 
Imbernón Muñoz, F., Jarauta Borrasca, B. y Medina Moya, J.L. (2010) "La enseñanza reflexiva en la educación superior", Cuadernos de docencia universitaria, $\mathrm{n}^{\mathrm{o}} 17$.

López Noguero, F. (2005) Metodología participativa en la enseñanza universitaria, Narcea, Madrid.

Medina Moya, J.L y Jarauta Borrasca, B. (2013) "La enseñanza como facilitación para el aprendizaje crítico reflexivo", en Enseñanza y aprendizaje en la educación superior, Síntesis, Madrid.

Quintero Olivares, G. (2010): La enseñanza del Derecho en la encrucijada. Derecho académico, docencia universitaria y mundo profesional, Civitas-Thomson Reuters, Navarra.

Viana Ballester, C. (2017) "Estrategias didácticas de aprendizaje colaborativo, comunicación oral y argumentación en derecho penal", en Innovación docente y ciencia jurídica. Aranzadi, Pamplona.

Villacañas Berlanga, J.L. y Castro Orellana, R. (2018) Foucault y la historia de la filosofía, Dado, Madrid. 